\title{
Protecting and Resolving Facial Skin from UV Rays and Air
} Pollution

Choi Yea Jin ${ }^{1}$, Thanida Laopanupong ${ }^{2 *}$

${ }^{1}$ Ekamai International School, Bangkok, Thailand.

${ }^{2}$ Department of Microbiology, Faculty of Science, Mahidol University, Bangkok, Thailand.

*Corresponding Author: Thanida Laopanupong, Department of Microbiology, Faculty of Science, Mahidol University, Bangkok, Thailand. Received date: January 22, 2021; Accepted date: February 12, 2021; Published date: February 16,2021

Citation: Choi Y Jin, T Laopanupong. (2021) Protecting and Resolving Facial Skin from UV Rays and Air Pollution. Journal of Clinical and Laboratory Research; 2(1); DOI:10.31579/2768-0487/003

Copyright: (C) 2021, Thanida Laopanupong, This is an open access article distributed under the Creative Commons Attribution License, which permits unrestricted use, distribution, and reproduction in any medium, provided the original work is properly cited.

\begin{abstract}
What is acne vulgaris? A commonly diagnosed skin disease concerning clogging and inflammation of pilosebaceous units is called acne vulgaris. Mostly, adolescence and puberty are affected because of hormone and their daily routine. Gender is one of the critical factors to cause acne. Thus, excellent skin cares are needed to prevent acne vulgaris, leading to another symptom, including stress, low self-esteem, and depression. Importantly, due to our surroundings, there are many kinds of pollution that we have to face in our daily lives and cannot be avoided. One of the causes of acne is air pollution; air pollution can come in many kinds, such as UV rays, smoke, or PM (particulate matter). PM carries out small particles in the air that clog our skin's pores or UV rays that directly damage our face if we do not have proper protection, resulting in acne outbreaks. Treating these acne outbreaks can be a lengthy and challenging process. Therefore, this review was to provide and update the method to shield and resolving the fair facial skin among air pollution surrounding. The author conducted this review thought collecting the article from Google Scholar and Pubmed using these keywords, including Acne vulgaris, air pollution, treatment, adolescence, self-esteem, and protection. There are a few steps that will protect and help resolve your skin from air pollution. First of all, cleansing your skin with a gentle cleanser is an excellent option to keep natural moisture in your skin. After cleansing, it is essential to use a gentle and soothing toner that helps remove grease and traces of dirt from blocking your pores, which might trigger acne. Scrubbing is the next step after the toner has been applied; mild scrubbing will ensure your skin is thoroughly cleansed of all harmful particles clogging your pores. Facial oil proves to boost moisten and shield your facial skin from toxic air substances. However, for long-term using facial oil can cause irritation to your skin, another option can be moisturizer instead. Make sure to apply sunscreen to protect against both UVB and UVA rays and apply it multiple times throughout the day when you are exposed to the air pollution. In addition, high sugary, salty and fats diets have the relationship with acne vulgaris. People with acne must avoid these types of food to reduce negative effect to acne. The protection and maintenance of facial skin is significantly important to make our face healthy. Moreover, the perks of having clear and healthy facial skin can boost up your confidence as well as decreasing our stress and anxiety that are being released throughout your body.
\end{abstract}

Keywords: Acne vulgaris; air pollution; emotional psychology; diet; cleanser; toner; moisturizer; facial oil; exfoliating; sunscreen and skin care

\section{Introduction}

A commonly diagnosed skin disease concerning clogging or inflammation of pilosebaceous units is Acne vulgaris [1]. Pilosebaceous units consist of the hair shaft, the hair follicle, the sebaceous gland, and the erector pili muscle [1]. Mostly, acne develops on the facial skin and often affect to adolescence and puberty [2]. There are many factors that cause acne to be developed on our skin, specifically facial skin [2]. The elements involve hormonal changes, cosmetics, emotional stress, menstruation, specific medication, dietary, and environmental pollution [3].

Environmental pollution can be categorized as air pollution, water pollution, soil pollution, sound pollution, radioactive pollution and thermal pollution. Among environmental pollution, air pollution is the most threatening to the living organism especially human [4]. Air pollution is a significant problem in our body, such as respiratory disease and skin disease [5]. Effect of air pollution on facial skin leads to acne outbreaks. It breaks down the skin collagen and the lipid layer, which have weakened the functions of the skin barrier [6]. Major air pollution involves PM 2.5, PM 10, $\mathrm{O}_{3}, \mathrm{CO}, \mathrm{NO}_{2}$ and $\mathrm{SO}_{2}$ [7]. In Thailand, PM 2.5 becomes the major air pollution many years [5]. The dirt particles, which are 200 times smaller than the pore diameter and therefore not visible to the human eye, cause the most harm to the skin [8]. Due to their size, pollution particles can infiltrate the deeper layers of the epidermis, which can stimulate inflammation and dehydration [8]. These damaging environmental agents have a very irritating effect, and once they penetrate 
the skin, they activate multiple pathways of inflammation, including melanocytes [9]. Furthermore, UV radiation is one of the significant environmental factors in air pollution, and when it comes to skin damage and aging, it is the most damaging pollutant among particulate matter [9]. Pollution has caused ozone layer to distress, which enhances the effects of free radicals on the skin and UV radiation [10]. UV rays induce the formation of free radicals in the skin, causing damage to the DNA and degradation of collagen and elastin in the skin, causing wrinkles to develop [11]. Moreover, it also triggers the development of melanin, which contributes to skin discoloration [11]. Melanin is a substance that gives pigment to the skin [11]. Non-melanoma skin cancers, including basal cell carcinoma (BCC) and squamous cell carcinoma, may result from the cumulative effect of sun exposure over time (SCC) [11]. UV exposure in early childhood may be a more critical factor in very lethal melanoma skin cancers. There are two forms of UV rays: UVA and UVB [12]. UVA rays are long, penetrating the deeper layer of the surface of the skin. Unprotected exposure from UVA rays leads to premature skin aging and wrinkles, as well as some skin cancers. UVB rays can penetrate the outlayer of the skin [12]. These rays have more energy than their UVA counterparts and are the cause of most skin cancers because they damage the skin cells' DNA directly.

Acne is most common among adolescence going through puberty, though it affects people of all ages [2]. During this period, your body undergoes regular changes. These factors may stimulate the production of oil, leading to an increased acne risk [2]. Puberty-related hormonal acne typically subsides, improving more or less as you reach puberty [13]. There are claims that there are certain risk factors for developing acne, including a diet rich in sugars and carbohydrates, or evolution, daily changes triggered by puberty or pregnancy, certain birth control pills or corticosteroids [13]. Men's skin is different than women's coats. It is more oily, larger pores, and can be more acne-prone [14]. Other hormones in men acne problems are not present in women, and some hormones found in women are not found in men either. Adolescent boys tend to have bad acne due to the abundance of sebum they produce [14]. Moreover, older men can also get acne triggered by a specific hormone called androgen [14]. Androgens are a group of chemically related sex steroid hormones that are mostly found in men than women [14]. Acne is more likely to occur in adolescence than men because of their hormone [15]. Males have higher concentration of androgens from puberty onwards, activating the oil production glands [15]. With more severe acne, men are more likely to suffer from acne scarring than women [15]. Men who still have acne as adults have usually been managing it since their teen years [15]. Adult men, nonetheless, they are less likely than their female counterparts to experience acne for the first time. The burden of adultonset acne is borne by women, and it is influenced by the changes in hormone levels that many women at certain times have [16]. This includes periods, pregnancies, and polycystic ovary syndrome [16]. Some women have acne breakouts just in time of their menstruation [16]. During pregnancy, numerous women have acne symptoms, usually during the first three months [16]. Polycystic ovary syndrome is a chronic condition that can trigger acne, weight gain, and the foundation of small cysts within the ovary [16].

There is a major concern about the psychological and social impacts of acne, primarily because acne affects teenagers at a critical time when they are building up their personalities [17]. During teenagers time, peer acceptance is essential for adolescents, and unfortunately, it's been discovered there are a significant correlations between physical appearance and appeal and peer status [17]. Several distressed patients with acne avoid eye contact, hide their face with their hair, and girls prefer to wear cosmetics to cover their breakouts, even after they have learned that makeup worsen their skin [17]. Facial acne provokes other adolescents taunts, and others find it difficult to find relationships [17]. When teenagers are learning to find connections, acne may be the barrier to make these bonds because it developed a lack of self-confidence [17]. Teenagers became shy and even reclusive because of the concern of others' negative judgment triggered by acne [18]. In severe cases, social phobia can be developed [18]. In education and work, acne may be the reason why patients with acne are less effective in applying for jobs [19]. Children with acne hesitate to go to school, which leads to poor academic performance [19]. Some patients with acne problems may result in depression; signs of depression include loss of appetite, mood irritation, spontaneous sobbing, and even feelings of unworthiness [19]. Depression can manifest in adolescents as social isolation (flee to the bedroom or peer exclusion) or disrupted school performance (lower grades or missed assignments) [19]. Extreme cases have resulted in suicide attempts and successful suicides [20]. Acne treatment, precisely isotretinoin, may be associated with depression [20]. There is a controversy about whether the treatment induces depression [21]. Depression, however, also occurs from acne and psychological disturbances stated above. If your acne is related to your life, seek support from your family doctor or dermatologist promptly [22]. Share all your issues to your doctor so that they can help you take control of your acne. Acne can be treated with medication, such as acne creams, lotions, or prescription medications [22]. An adolescent's anxiety over their acne must be managed appropriately.

Air pollution is a significant problem in every area $[4,6,9,11]$. Air pollution is the leading cause of acne outbreaks [9]. It collapses the collagen and the lipid layer on the skin, affecting the functions of the skin barrier [9]. With growing pollution, there are a few anti-pollution steps that can be taken to maintain and restore the health of your skin [23]. Firstly, a two-step process for cleaning is suggested [24]. To remove any excess make-up, dirt, or pollution left on the skin, you can start by using a cleaning wipe or make-up removal pad and then rinse with a cleanser [24]. It is recommended to use cleansing brushes, which are more effective than using hands [25]. Secondly, clam and soothing facial toner is good choices to adjust your face ph [26]. Thirdly, once toner was applied, scrubbing is optional strategy to boost your facial health [27, 28]. Next, a serum or moisturizer containing an antioxidant can be used to help create protective barrier and prevent small particles from penetrating into the skin [29, 30]. Lastly, applying sunscreen is a must because it creates layers of protection against the UV lights and traps the smog particles from harmful chemicals that try penetrating deep into the skin [31-33]. Additionally, an excellent approach is an increase in the consumption of citrus fruits, pomegranate, berries, carrots, and green tea that are loaded with antioxidant energy [34, 35]. It is best to switch from alcoholic drinks to red wine which is packed with polyphenol-based antioxidants [36, 37]. In this review, we provide the protection and regenerating method for the facial skin due to air pollution damage. Moreover, with the rising of the air pollution level, the demand for antipollution skin care is gradually needed and an interesting area for new research.

\section{Cleansers}

Normally, the skin has protective mechanisms that help resist free radicals in contaminated air through secreting fats and oils [38]. The dried-out skin combined with deadly contaminants free-flowing in the air due to high acidic air temperatures, leading to the skin being susceptible to the intrusion of toxic chemicals [38]. The skin needs to be kept moisturize. Therefore, using a gentle cleanser is a good selection [39, 40]. Look for a cleanser with a soft and non-aggressive to sensitive skin, or moisturizing cleanser that will strengthen and not strip the skin natural barrier [24, 40]. For multiple facial skin forms, there are several kinds of cleansers available for purchase with special formulations and healthy skin benefits [40, 41]. Starting with a water-based cleanser, this cleanser is popular among teenagers [42]. Some water-based cleaners do not contain oilbased ingredients [42]. They do not leave a greasy, shiny feel like other cleansers. Therefore, they can be overly dry and cause a mattifying effect 
on dry skin or excessive oiliness in acne-prone skin. This type of cleanser is a recommended choice for oily and acne-prone skin types. However, if you have dry skin, make sure that your water-based cleanser has hydrating ingredients that will not strip away your skin moisture. Next, the oil-based cleansers are formulated with a suitable type of oil that remove harmful particles from the skin [42]. When you wash your face with an oil-based cleanser, you are rinsing away the excessive fat, leftover makeup, and impurities that your facial skin clings on. Next is the gel cleansers that have similar benefits to cleaning oil [42]. To get rid of extra oil and eliminate damaging contaminants from the pores, it utilizes oil-based ingredients. They are slightly more soothing than oil cleansing. This helps water and oil to connect with each other in both the face wash and your skin without stripping the natural oil of your skin and also achieving a thorough cleaning. Both of the oil cleansers and gel cleansers are suitable for oily and acne-prone skin. Moving on to foam cleansers, it is the lightest face wash among all types of cleaners [42]. When you pump a foam cleanser into your hands, it may start as a gel or cream, but as you lather it up, the texture becomes an airy foam. They can be oil-free or contain nourishing oils. It can be beneficial for oily skin and those with acne prone. Lastly, cream cleansers are well known as a moisturizer, but they can be hydrating and nutritious [42]. They included antibacterial properties that strip away dirt, unclog pores, and deliver moisture to dehydrated skin. This cleanser is best suited for dry and sensitive skin since it would not leave skin feeling rough and tight after cleansing [42]. The double cleansing strategy will help ensure that any breakout inducing irritants, debris, and makeup on your facial skin is completely washed away [43, 44]. Moreover, a double cleanser is a smart cleaning strategy, but it is warned that using the same cleaner for both stages 1 and 2 is unsuitable [44]. This is one of the options for cleansing your face to fight off pollution. To help break down pollutants and cut through the build-up of grime, start with an oil-based cleanser, then use a cream cleanser to thoroughly clean and remove pollution particles that cling to the skin.

\section{Toners}

It is necessary to neutralize it with the proper toner after washing your skin. A soft, calming toner allows the skin to extract oil and remnants of dirt and grime, ensuring that there are no acidic particles remaining [45]. Since cleansers causes the $\mathrm{pH}$ of the skin to be too acidic, so toner helps toning it down $[46,47]$. It's exceptionally effective for acne-prone skin. A toner is a convenient, absorbing liquid that helps in removing excess dirt, oil traces, makeup, and correct and balance the $\mathrm{pH}$ of the skin, bringing down the acidic $\mathrm{pH}$ to around 5.5 [48]. There are three types of toner, which are hydrating toners, exfoliating toners, and treatment toners $[49,50]$. To give the skin a rapid improvement in hydration and refreshment, hydrating toners are used [51]. This toner contains moisturizers such as hyaluronic acid or glycerin that remove the sensation of tightness [52]. They do not perform much with deep cleansing. However, they put on an extra hydration layer. Hydrating toners are suitable for all skin types: normal, combination, oily, and especially for dry skin [52]. This extra layer of hydration will normalize the skin and plump it up right after cleansing. Next up is exfoliating toners. They typically contain a type of hydroxy acid or fruit enzymes [53]. The most popular types of exfoliating toners are the ones with glycolic acid toners and salicylic acid toners [53-55]. Furthermore, it gets rid of dead skin cells, and it also boosts collagen production and increases the dividing of new skin cell. Toners that can exfoliate keep the pores clean and decrease their visibility. They are ideal for types of mild, mixed, and oily skin because they can irritate dry skin and sensitive skin. Hence, if super gentle exfoliating acids are used, dry and sensitive types of skin will benefit as well. The toners are lightly exfoliated with lactic acid or poly-hydroxy acids $[55,56]$. As it can hydrate and resist cracking of the skin, they are usually more tender on the skin. Finally, treatment toners, such as acne caused by air pollution, are more focused and created to manage actual skin conditions [57]. They are usually marketed as soothing toners, brightening toners, anti-aging toners, balancing toners and etc [58, 59]. They do more than moisten the skin since they contain potent active ingredients in the brightening toner to get rid of discoloration, such as vitamin C. Similarly, to eliminate inflammation and redness of the skin, a calming toner would provide soothing herb extracts such as chamomile or allantoin [59]. To neutralize wrinkle-causing free radicals, an antiaging toner might contain antioxidants [60]. Treatment toners are effective for all skin types, depending on the problem, to target skin conditions such as excess oil, large pores, scarring, irritation, or air pollution. The study of toner with facial skin is minimal. However, some studies reported that natural extracts such as guava and tea tree, improved the facial skin problem with oily skin type [46, 57]. Thus, a toner containing these natural extracts may help to resolve the facial skin from air pollution.

\section{Facial oil and moisturizer}

Do you know the differences between using a facial oil or a moisturizer? Which one is better? Moisturizing your face is essential [29]. Not only does it help achieve youthful skin, but it plays a vital role in serving as a barrier from pollution, helps remain youthful skin, and reduces acne and blemishes on the skin. Facial oil is emollient-rich to give the deep-skin nourishment [61]. They also nourish and restore your natural glow on the skin while providing you with long-lasting hydration. Facial oils offer plenty of essential fatty acids and vitamin $C$ that are good for your skin [61]. They help to re-hydrate skin, dissolve grime, and they are rich in vitamins and antioxidants. Facial oil also helps draw the active ingredients in your other skin products to absorb deep into the skin, and they are a great help to calming the skin against any harsh elements. They are best recommended in small amounts, too much oil can clog your pores. There are two types of facial oils: essential and vegetable oils [61]. Essential oils are high concentrations of natural plant oils and carrier, while vegetable oils are used to dilute essential oils. When they are combined, they work together to penetrate the harmful particles deep into the skin. When applying the facial oil, be cautious not to rub the oil into the skin. The best method to using it is to warm a few drops in your hands and lightly press your hands to your face. The heat of your hands will help the oil absorb more in-depth into the skin layer. Regardless, long-term use of facial oil (especially oil rich in oleic acid, such as olive or argan oil) has been shown to be capable of destroying the skin barrier [62]. Oleic acid can deliberately weaken and break down the skin barrier, while fat inhibits self-repair of the skin barrier in general [63]. The number of skin cells that contain fats in the lipid layer is significantly decreased as we aged. Therefore, with time, the skin layer gradually weakens, and inflammation grows as the skin gets worse as it defends itself from bacteria and contaminants. As our collagen and elastin are being damaged by inflammation, the skin starts aging more rapidly [64]. You do damage to the skin by over-using oil by hindering the ability of the skin to rebuild the skin barrier and by accelerating the deterioration of the proteins that make the skin youthful- collagen and elastin. Therefore, it is recommended to use linoleic oils instead of oleic oils since they are much lightweight and less vague, they can always be used with caution $[61,65]$. This is a sign that you need a rest from facial oils if you ever feel like the skin is both dry and greasy, or that oils do not penetrate well into the skin no matter how little you use and how much you rub and leave the oily surface layer. A prominent facial oil, as long as the strong foundation is controlled, it will also contain oils rich in oleic acid. On the other hand, facial moisturizers are made with water oil and other essential ingredients [43, 51]. Moisturizers hydrate the epidermis, meaning the uppermost layer of the skin. They have skin-replenishing ingredients such as hyaluronic acid, ceramides, glycerin, petrolatum, salicylic acid and so on $[29,59]$. Moisturizer is a balanced combination of both oil and watersoluble nutrients. There are four types of moisturizers: humectants, emollients, ceramides, and occlusives [29, 59]. Humectants moisturizers can remove water from the skin cells in order to keep the skin hydrated 
[66, 67]. The vital ingredients to it are glycerin, lactic acid, urea, hyaluronic acid, and AHAs. Humectants serve as an excellent first coat of moisture and are effective because they are free of the heavy oils for all skin types including dry, combinations, and acne-prone skin. The next moisturizers are the emollients which are the ones that fill in some gaps or holes among skin cells that lack moisturizing lipids $[67,68]$. The skin comes to be stronger and hydrated easier. The key ingredients include lanolin, mineral oil, and petrolatum. For dehydrated, aged skin that requires significant moisture, this works best. Moving on to ceramides moisturizers, these are normally contained in the skin [69]. Damaged or low ceramide levels appear as dry damaged skin [69]. It will preserve moisture by replenishing the skin with missing ceramide. This works best with skin types that have minimal dryness and those of eczema, rednessrash of the skin, both normal and combination. Lastly, the occlusives moisturizers that are designed to douse the skin in moisture $[67,68]$. They are weighty and are highly recommendable for the body instead of the face. The ingredients include rosehip, avocado, and hazelnut oils [70]. It is most effective for more aged, dehydrated skin that is deprived of all humidity in the environment. It's safe to stop this moisturizer if the skin is allergic or blemish prone.

\section{Scrubbing}

Once the toner is evenly applied, gently rinse your skin. Face scrub should be used on any areas of the body that have been exposed to sunlight. Each day, a 10-second scrub allows the skin to regenerate and heal from the ill effects of pollutants [71]. A micro-exfoliation cream and a boost of extra hydration should be applied a couple days a week to help further heal the skin from the daily environmental influences that attack its fragile surface [72]. Nevertheless, cleansing alone is not enough, exfoliating is needed. By eliminating your dead skin cells does not make your skin more tender but the very opposite happens by making the deep dermal layer of the skin thicker and creating a greater barrier to air pollutants, it is less likely to grow fine lines and wrinkles. There are two main methods of exfoliating: physical and chemical [73]. Physical exfoliants are manually brushed off dead cells on the facial skin by using particulates or granules or tools such as cleansing brushes [73, 74]. For oily and dry skin types, face scrubs are usually much better because they whisk away dry skin spots and shift skin texture drastically $[73,74]$. Cleansing brushes such as sonic facial brush is an excellent tool with rotating bristled heads that helps clean and polish your skin while using a good facial wash [75]. Depending on your skin type, use the brush anywhere from once or twice a week to daily uses. Either way, make sure to regularly clean and disinfect the brush head. A cleansing scrub delivers a smoother and more thorough cleanse than simply using a washcloth or the fingertips. The bristles dig into the skin surface deeper, getting rid of more gravel, dust, and grease. On the other hand, chemical exfoliating is done by using chemicals such as salicylic acid and retinoids $[73,74]$. When these ingredients are applied to the skin, the bond between dead skin cells is subtly destroyed, so they can be dislodging. This also helps remove any pollution that is trapped between the dead skin cells at the same time. Chemical exfoliators are not only gentler and more effective than physical formulas, but they also come in two different forms depending on what your skin needs. Essentially, there are two types of chemical exfoliants: alpha hydroxy acid (AHA) and betahydroxy acid (BHA) [73, 74]. Alpha hydroxy acids (AHAs) are watersoluble, meaning they exfoliate the surface of your skin, drawing moisture while they work to keep your face hydrated [76]. They are also effective at loosening dead cells to make skin brighter and smoother with daily usage. AHAs come in a few forms, like lactic, mandelic, glycolic, and tartaric acid. If you have sensitive skin or are new to chemical exfoliants, start with lactic acid, which tends to be the lightest of AHAs. If you have a standard skin type with no real sensitivity issues, try glycolic acid since they are a bit stronger and faster acting. AHAs are best for normal, dry, sensitive, or redness-prone skin. BHAs are oil-soluble, meaning they break down oil clogged pores to treat blackheads, whiteheads, and zits
[77]. They are also anti-inflammatory, meaning they will help mitigate some of the irritating effects that BHAs can cause. Salicylic acid is the only one common BHA that helps with acne spot treatment. If your skin is super tough, you can use your BHA interchangeably with AHAs, but they tend to be irritating and drying if used in high concentrations over the entire facial skin. Start with only one chemical exfoliant at a time, and use BHA every three nights on clean, dry skin, waiting at least 10 minutes before applying the rest of the other skincare $[53,77]$. And as a final note, it is essential to know that many chemical exfoliants increase your skin sun sensitivity. Be sure to apply and reapply sunscreen throughout the day.

\section{Sunscreen}

From UV rays to PM 2.5 and pollution, these environmental factors function by causing oxidative damage to the skin surface, which breaks down collagen and thus affects the skin barrier function $[6,9,23]$. An impaired skin layer renders it to be vulnerable to air pollution toxicity. $\mathrm{PM}$ is a combination of small particles such as organic compounds and dirt or ashes, as well as liquid droplets. Skin barrier functions can be seriously damage and reactions, including immune dysregulation, activation of melanocytes, and collagen breakdown, caused by the highlevel PM 2.5 exposure $[6,9,23]$. However, there is a way to protect the skin from both UV rays. Most sunscreens are traditionally used for protecting only against UVB rays [78]. In contrast, a double-duty SPF can protect against both UVB and UVA [33, 78]. Make sure to slather it on multiple times throughout the day. Additionally, choosing physical sunscreens, typically made with mineral-based formulas, are thought to be better for sensitive skin and may contain fewer skin irritants than chemical sunscreens [31]. Sunscreen could be a great way to not only shield your skin from UV exposures but also to moisturizing. Look for sunscreens that contain hydrating ingredients, such as hyaluronic acid or glycerin [79]. If you prefer natural ingredients, look for aloe vera or honey containing in sunscreens [33]. This is recommended especially for dry and sensitive skin. Oily skin type is suitable for water-based sunscreens, and there are many products on shelves labeled "ultra-light" [33]. Look for sunscreens that contain tea tree oil or niacinamide to keep your skin natural production of oil under control $[33,78]$. Acne-prone skin is ideal for mineral sunscreen, with zinc oxide or titanium dioxide as the active, protecting ingredients [80]. As with both acne-prone and oily skin, try finding a water-based product and feels light for your skin to be able to breathe without clogging the pores. SPF stands for Sun Protection Factor, and it is extremely important to pick a sunscreen with high SPF level [81]. Sunscreen with an SPF of at least 30, which blocks 97 percent of the sun's UVB rays, is recommended among dermatologists [81]. Higher numbers of SPFs protect significantly more UVB rays from the sun, but no sunscreen will block 100 percent of the UVB rays from the sun. SPF offers your skin considerable advantages, particularly protecting it from UV rays that cause wrinkles, loss of firmness, dullness, and uneven pigmentation [9]. Products with additional SPF have been shown to help avoid signs or symptoms of aging when used daily.

\section{Dietary and acne}

Cleansing and applying sunscreen are some of the many methods of having clear skin. Dietary control is an indirect way of having a clear coat if you do not prefer using chemicals on your skin [82]. What you eat significantly affects your overall body functions, especially your skin. The biggest takeaway from recent diet and acne studies is that a lowsugar, well-balanced diet is ideal for inflammation reduction and hormone regulation [82]. Foods are digested and divided into vitamins, minerals, and amino acids that can be used by your body to encourage better skin [83]. However, if you do not ingest sufficient protein, the amino acids that are used to make collagen and elastic tissue are forced to remove from your skin. Be sure to avoid eating processed food and refined sugar since 
these causes the blood sugar levels rising very fast rate, and the pancreas respond through releasing insulin [82]. By eliminating sugar, it is possible to decrease the amount of insulin the body produces [82]. Cutting out dairy products and alcohol is also crucial since milk contains precursors to testosterone and other androgens that affect the skin's hormone receptors to activate the acne-causing process [34, 35, 37]. A lowglycemic diet is recommended to improve the skin. The glycemic load (GL) is a measure of the type and amount of carbohydrates that you take $[34,35,37]$. It is recommended that you keep your daily GL below 100 when following the low GL diet. Foods high in glycemic foods and beverages results in high blood sugar, such as white bread, corn flakes, puffed rice, potato chips, white potatoes or fries, doughnuts or other pastries, sugary drinks such as milkshakes and white rice should be avoided at all cost $[34,37]$. Most vegetables, some fresh fruits, beans, and steel-cut oats are included in low-glycemic diets. A decrement in weight and cholesterol has been affected by low GL diets. High GL diets, on the other hand, have been related to respiratory disease and an elevated risk of several cancers. In the USA, 2,258 patients were put on a low-glycemic diet that improved their acne, according to the study, with 87 percent of patients reporting they had less acne and 91 percent saying they require less prescription for acne [84]. In addition, 32 patients with acne in Korea consumed either their regular diet or a low glycemic diet for 10 weeks [82]. Those who adopted the low-glycemic diet had slightly less acne than those who consumed a normal diet at the end of 10 weeks [82]. Since this diet reduces elevation of blood sugar, practicing a low-glycemic diet will contribute to less breakouts. It induces inflammation around the body as the blood sugar increases; these spikes are responsible for helping your body create more sebum in your skin. Inflammation and excess sebum can also contribute to facial acne.

\section{Conclusion}

With the rise of air pollution among us, including UV rays, PM 2.5 and etc. Acne-prone skin results from exposure to these pollutants. Therefore, a proper skincare routine is essential to protect and regenerate our healthy skin. There are five steps to achieving clear skin in a polluted environment: cleansing, toning, scrubbing, facial oil or moisturizing, and sunscreen, which must improve acne-prone skin thought out air pollution. The research about the relationship between acne and air pollution is minimal. Regardless, the problem of air pollution is still increasing daily. Moreover, acne affects people self-esteem, confidence, and cause severe depression, mostly in teenager and puberty. Benefit of acne-clear skin increases our self-esteem, at the same time, decreases both our selfanxiety and depression. Importantly, it makes our face stronger in the exposed pollution.

\section{Reference}

1. Williams, H.C., R.P. Dellavalle, and S. Garner, Acne vulgaris. (2012) The Lancet, 379(9813): p. 361-372.

2. Lynn, D.D., et al. (2016) The epidemiology of acne vulgaris in late adolescence. Adolescent health, medicine and therapeutics.

3. Dréno, B., et al. (2018) The influence of exposome on acne. Journal of the European Academy of Dermatology and Venereology. 32(5): p. 812-819.

4. Vierkötter, A. and J. (2012) Krutmann, Environmental influences on skin aging and ethnic-specific manifestations. Dermato-endocrinology. 4(3): p. 227-231.

5. Vichit-Vadakan, N., et al. (2001) Air pollution and respiratory symptoms: results from three panel studies in Bangkok, Thailand. Environmental Health Perspectives. 109(suppl 3): p. 381-387.

6. Kim, K.E., D. Cho, and H.J. (2016) Park, Air pollution and skin diseases: Adverse effects of airborne particulate matter on various skin diseases. Life sciences. 152: p. 126-134.
7. Nagaraj, S. and R.V. Biradar. (2017) Applications of wireless sensor networks in the real-time ambient air pollution monitoring and air quality in metropolitan cities - a survey. in International Conference On Smart Technologies For Smart Nation (SmartTechCon). 2017. IEEE.

8. Dulog, R.C., et al. (2000) Method for removing dead surface cells, dirt, oil, and blackheads from the skin and related compositions and articles. Google Patents.

9. Schikowski, T. and A. Hüls. (2020) Air pollution and skin aging. Current Environmental Health Reports. p. 1-7.

10. Bais, A.F., et al. (2018) Environmental effects of ozone depletion, UV radiation and interactions with climate change: UNEP Environmental Effects Assessment Panel, update 2017. Photochemical \& Photobiological Sciences. 17(2): p. 127-179.

11. Pillai, S., C. Oresajo, and J. Hayward. (2005) Ultraviolet radiation and skin aging: roles of reactive oxygen species, inflammation and protease activation, and strategies for prevention of inflammation-induced matrix degradation-a review. International journal of cosmetic science. 27(1): p. 1734.

12. Wlaschek, M., et al. (2001) Solar UV irradiation and dermal photoaging. Journal of Photochemistry and Photobiology B: Biology. 63(1-3): p. 41-51.

13. Thiboutot, D. (2004) Acne: hormonal concepts and therapy. Clinics in dermatology. 22(5): p. 419-428.

14. Goulden, V., G. Stables, and W. Cunliffe. (1999) Prevalence of facial acne in adults. Journal of the American Academy of Dermatology. 41(4): p. 577-580.

15. Knaggs, H., et al. (2004) Post-adolescent acne. International journal of cosmetic science. 26(3): p. 129-138.

16. Tanghetti, E.A., et al. (2014) Understanding the burden of adult female acne. The Journal of clinical and aesthetic dermatology. 7(2): p. 22.

17. Van der Meeren, H., W. Van der Schaar, and C. Van den Hurk. (1985) The psychological impact of severe acne. Cutis. 36(1): p. 84 .

18. Krejci-Manwaring, J., et al. (2006) Social sensitivity and acne: the role of personality in negative social consequences and quality of life. The International Journal of Psychiatry in Medicine. 36(1): p. 121-130.

19. Gollnick, H.P., A.Y. Finlay, and N. Shear. (2008) Can we define acne as a chronic disease? American journal of clinical dermatology. 9(5): p. 279-284.

20. Purvis, D., et al. (2006) Acne, anxiety, depression and suicide in teenagers: A cross-sectional survey of New Zealand secondary school students. Journal of paediatrics and child health. 42(12): p. 793-796.

21. Marqueling, A.L. and L.T. Zane. (2005) Depression and suicidal behavior in acne patients treated with isotretinoin: a systematic review, in Database of Abstracts of Reviews of Effects (DARE): Quality-assessed Reviews [Internet]. Centre for Reviews and Dissemination (UK).

22. Smithard, A., C. Glazebrook, and H. Williams. (2001) Acne prevalence, knowledge about acne and psychological morbidity in mid-adolescence: a community-based study. British Journal of Dermatology. 145(2): p. 274-279.

23. Rembiesa, J., et al. (2018) The impact of pollution on skin and proper efficacy testing for anti-pollution claims. Cosmetics. $\mathbf{5}(1): \mathrm{p} .4$.

24. Isoda, K., et al. (2015) Efficacy of the combined use of a facial cleanser and moisturizers for the care of mild acne patients with sensitive skin. The Journal of dermatology. 42(2): p. 181-188. 
25. Schnabl, D., et al. (2020) Cleansing efficacy of waist-shaped inter-dental brushes. A randomized-controlled crossover study. Journal of Clinical Periodontology. 47(1): p. 30-35.

26. Wilson, R.F. (2011) Enlarging the Regulation of Shrinking Cosmetics and Sunscreens.

27. Street, V.D. (2000) Cosmetic method for removing detritus and foreign matter from the epidermis and a cosmetic abrasive pad for scrubbing the epidermis. Google Patents.

28. Kwon, K.C., et al. (2021) Reduction of enlarged facial pore using ion-paired amino acid through enhancement in skin permeation and exfoliation: A placebo-controlled in vivo study. Journal of cosmetic dermatology.

29. Draelos, Z.D. (2018) The science behind skin care: moisturizers. Journal of Cosmetic Dermatology. 17(2): p. 138144.

30. Han, S.M., et al. (2015) The beneficial effects of honeybeevenom serum on facial wrinkles in humans. Clinical interventions in aging. 10: p. 1587.

31. English, J., I. White, and K. Cronin. (1987) Sensitivity to sunscreens. Contact dermatitis. 17(3): p. 159-162.

32. $\mathrm{Xu}, \mathrm{S}$., et al. (2016) Sunscreen product performance and other determinants of consumer preferences. JAMA dermatology. 152(8): p. 920-927.

33. Donglikar, M.M. and S.L. Deore. (2016) Sunscreens: A review. Pharmacognosy Journals. 8(3).

34. Bowe, W.P., S.S. Joshi, and A.R. Shalita. (2010) Diet and acne. Journal of the American Academy of Dermatology. 63(1): p. 124-141.

35. Kucharska, A., A. Szmurło, and B. Sińska. (2016) Significance of diet in treated and untreated acne vulgaris. Advances in Dermatology and Allergology/Postẹpy Dermatologii i Alergologii. 33(2): p. 81.

36. Claudel, J.-P., et al. (2018) Acne and nutrition: hypotheses, myths and facts. Journal of the European Academy of Dermatology and Venereology. 32(10): p. 1631-1637.

37. Spencer, E.H., H.R. Ferdowsian, and N.D. Barnard. (2009) Diet and acne: a review of the evidence. International journal of dermatology. 48(4): p. 339-347.

38. Poulsen, F. and P. Samuelsen. (1983) Skin barrier., Google Patents.

39. Dall'Oglio, F. et al. (2014) Cosmetics for acne: indications and recommendations for an evidence-based approach. Giornale italiano di dermatologia e venereologia: organo ufficiale, Societa italiana di dermatologia e sifilografia. 150(1): p. 1-11.

40. Mukhopadhyay, P. (2011) Cleansers and their role in various dermatological disorders. Indian journal of dermatology. 56(1): p. 2.

41. Toombs, E.L. (2005) Cosmetics in the treatment of acne vulgaris. Dermatologic clinics. 23(3): p. 575-581.

42. Draelos, Z.D.(2018) The science behind skin care: Cleansers. Journal of Cosmetic Dermatology. 17(1): p. 8-14.

43. Nash, J.F., P.J. Matts, and K.D. Ertel. (2007) Maintenance of healthy skin: cleansing, moisturization, and ultraviolet protection. Journal of Cosmetic Dermatology. 6: p. 7-11.

44. Kottner, J., A. Lichterfeld, and U. Blume-Peytavi. (2013) Maintaining skin integrity in the aged: a systematic review. British Journal of Dermatology. 169(3): p. 528-542.

45. Draelos, Z.K. (1995) Cosmetics: an overview. Current Problems in Dermatology. 7(2): p. 45-64.

46. Pongsakornpaisan, P, N. Lourith. (2019) and M. Kanlayavattanakul, Anti-sebum efficacy of guava toner: A split-face, randomized, single-blind placebo-controlled study. Journal of Cosmetic Dermatology. 18(6): p. 1737-1741.

47. Rivera, J., What Skin Toner Does And Why You Should Care.
48. Schreml, S., M. Kemper, and C. Abels. (2014) Skin pH in the elderly and appropriate skin care. Eur Med J Dermatol. 2: p. 8694.

49. Sharma, S., Skin pH-Why It Matters More than You Think Skin $\mathrm{pH}$-Why It Matters More than You Think.

50. anghvi, A.S., Homemade Skin Toners That Do Not Dry Out Your Skin.

51. Yuanxi, L., et al. (2016) Comparison of skin hydration in combination and single use of common moisturizers (cream, toner, and spray water). Journal of cosmetic science. 67(3): p. 175-183.

52. Selly, A., Effective Skin Care for Women.

53. Goodwin, B., Alpha, Beta, Enzymes: The Science of Chemical Exfoliation.

54. Barker, M.O.(1998) Masks and astringents/toners. Textbook of Cosmetic Dermatology. p. 155.

55. Ashford, A. (2020) The Effect of Skin Care Products Containing Salicylic Acid, Glycolic Acid, Hyaluronic Acid, and Rosa Damascena Extract on the Concentration of Micrococcus luteus.

56. Tung, R.C., et al. (2002) The Treatment of Rosacea with Glycolic Acid. BASIC AND CLINICAL DERMATOLOGY. 22: p. 37-52.

57. Timudom, T., et al. (2020) Anti-Sebum Efficacy of Phyllanthus emblica L.(Emblica) Toner on Facial Skin. Applied Sciences. 10(22): p. 8193.

58. Jensen, C.J. and H. Robinson. (2006) Methods for manufacturing an enhanced cosmetic skin care toner. Google Patents.

59. Johnson, R.M. (2013) Skin-Friendly Skin Care: Make Your Own Cleansers, Moisturizers, and Toners. BalboaPress.

60. Sumit, K., et al. (2012) Herbal cosmetics: used for skin and hair. Inven. J. p. 1-7.

61. Diana Draelos, Z., H. Gunt, and S.B. Levy. (2020) Naturebased botanical facial oil oxidative stress protection. Journal of Cosmetic Dermatology.

62. Bissett, D., et al. (2004) Topical niacinamide reduces yellowing, wrinkling, red blotchiness, and hyperpigmented spots in aging facial skin 1. International journal of cosmetic science. 26(5): p. 231-238.

63. Rabasco Álvarez, A.M. and M.L. González Rodríguez. (2000) Lipids in pharmaceutical and cosmetic preparations. Grasas y Aceites, 51 (1-2), 74-96.

64. Farage, M., et al.(2008) Intrinsic and extrinsic factors in skin ageing: a review. International Journal of Cosmetic Science. 30(2): p. 87-95.

65. Koh, K., et al. (2002) Tea tree oil reduces histamine-induced skin inflammation. British Journal of Dermatology. 147(6): p. 1212-1217.

66. Kraft, J. and C. Lynde. (2005) Moisturizers: what they are and a practical approach to product selection. Skin Therapy Lett. 10(5): p. 1-8.

67. Lynde, C. (2001) Moisturizers: what they are and how they work. Skin Therapy Lett. 6(13): p. 3-5.

68. Lipozenčić, J., Z. Paštar, and S. Marinović-Kulišić, Moisturizers. (2006) Acta Dermatovenerologica Croatica. 14(2).

69. Imokawa, G. (2002) Ceramides as Natural Moisturizing Factors. Skin moisturization. p. 267.

70. Sethi, A., et al. (2016) Moisturizers: the slippery road. Indian journal of dermatology. 61(3): p. 279.

71. Dhar, S. ( 2007) Newborn skin care revisited. Indian Journal of Dermatology. 52(1): p. 1. 
72. Merinville, E., et al. (2009) Exfoliation for sensitive skin with neutralized salicylic acid? International Journal of Cosmetic Science. 31(3): p. 243-244.

73. Leung, J., The Science of Exfoliants: Physical vs Chemical.

74. Draelos, Z.D. (2010) Cosmeceuticals off the face, in Body rejuvenation. Springer. p. 227-232.

75. Brewer, G.K., et al. (2013) Brushhead for electric skin brush appliance. Google Patents.

76. Clark 3rd, C. (1996) Alpha hydroxy acids in skin care. Clinics in plastic surgery. 23(1): p. 49-56.

77. Ramos-e-Silva, M., et al. (2001) Hydroxy acids and retinoids in cosmetics. Clinics in dermatology. 19(4): p. 460-466.

78. Ngoc, L.T.N., et al. (2019) Recent Trends of Sunscreen Cosmetic: An Update Review. Cosmetics. 6(4): p. 64.

79. Kwon, S.B., et al. (2013) The effect of glycerin, hyaluronic acid and silicone oil on the hydration, moisturization and transepidermal water loss in human skin. Asian Journal of Beauty and Cosmetology. 11(11): p. 761-768.
80. Yari, R., et al. (2018) Influences of combining nano zinc, honey and Aloe vera to accelerate healing the wounds caused by thirddegree burn in male balb/c mice. Journal of Basic Research in Medical Sciences. 5(1): p. 38-46.

81. Faurschou, A. and H. Wulf. (2007) The relation between sun protection factor and amount of suncreen applied in vivo. British Journal of Dermatology. 156(4): p. 716-719.

82. Jung, J.Y., et al. (2010) The influence of dietary patterns on acne vulgaris in Koreans. European Journal of Dermatology. 20(6): p. 768-772.

83. Wolf, R., H. Matz, and E. Orion. (2004) Acne and diet. Clinics in dermatology. 22(5): p. 387-393.

84. Szabó, K. and L. Kemény. (2011) Studying the genetic predisposing factors in the pathogenesis of acne vulgaris. Human immunology. 72(9): p. 766-773.
This work is licensed under Creative Commons Attribution 4.0 License

\section{To Submit Your Article Click Here: Submit Manuscript}

DOI: $10.31579 /$ jclr.2021/003.
Ready to submit your research? Choose Auctores and benefit from:

* fast, convenient online submission

* rigorous peer review by experienced research in your field

* rapid publication on acceptance

* authors retain copyrights

* unique DOI for all articles

* immediate, unrestricted online access

At Auctores, research is always in progress.

Learn more www.auctoresonline.org/journals/journal-of-clinical-andlaboratory-research 